\title{
WEAK SOLUTIONS OF DEGENERATED QUASILINEAR ELLIPTIC EQUATIONS OF HIGHER ORDER
}

\author{
PAVEL DRÁBEK \\ Department of Mathematıcs, Unıversity of West Bohemıa \\ Amerıcká 42, 30614 Plzeň, Czech Republıc
}

ALOIS KUFNER

Mathematical Institute, Czech Academy of Sciences Žıtná 25, 11567 Praha, Czech Republıc

FRANCESCO NICOLOSI

Dıpartımento dı Matematıca, Unıversıtà dı Catanıa Viale A Dorıa 6, 95125 Catanıa, Italy

(Received September 26, 1994 and in revised form July 11, 1995)

\begin{abstract}
We prove the exıstence of weak solutions of higher order degenerated quasilınear ellıptıc equations The main tools are the degree theory for generalızed monotone mappings and imbedding theorems between weighted Sobolev spaces The straightforward use of these imbeddings allows us to consider more general assumptions than those in our preceding paper [3]
\end{abstract}

Key Words And Phrases: Degenerated problems, quasilinear ellıptıc equations of higher order, weighted Sobolev spaces, degree of a mapping

AMS (MOS) Subject Classification Codes: 35J70, 35J60, 35J40, 26D10, 46E35, 47H1 1

\section{Introduction}

In the paper DRÁBEK, KUFNER and NICOLOSI [3], the problem of the existence of solutions of boundary value problems for higher order nonlinear degenerated elliptic equations was investigated. The underlying differential operator of order $2 m(m \geq 1)$ was supposed to be in the divergence form

$$
(A u)(x)=\sum_{|\alpha| \leq m}(-1)^{|\alpha|} D^{\alpha} A_{\alpha}\left(x, u(x), \ldots, D^{m} u(x)\right), x \in \Omega \subset \mathbb{R}^{N},
$$

and the weak solutions have been sought in a special weighted Sobolev space

$$
W^{m, p}(\nu, \Omega)
$$

$\left(1<p<\infty, \nu=\left\{\nu_{\alpha},|\alpha|=m\right\}\right.$ denotes a collection of weight functions), normed by

$$
\left(\sum_{|\alpha|=m} \int_{\Omega}\left|D^{\alpha} u(x)\right|^{p} \nu_{\alpha}(x) d x+\sum_{|\alpha|<m} \int_{\Omega}\left|D^{\alpha} u(x)\right|^{p} d x\right)^{\frac{1}{p}} .
$$

The weight functions $\nu_{\alpha}=\nu_{\alpha}(x)$ which describe in a certain sense the degeneracy or singularty of the operator $A$ from (1.1) appear only on the highest ( $m$-th order) derivatives. The assumptions

$$
\nu_{\alpha} \in L_{l o c}^{1}(\Omega), \nu_{\alpha}^{-\frac{1}{p-1}} \in L_{l o c}^{1}(\Omega)
$$

guarantee that the space $W^{m, p}(\nu, \Omega)$ is well defined and contains $C_{0}^{\infty}(\Omega)$ as a subset.

In addition to (1.3) the assumption

$$
\nu_{\alpha}^{-1} \in L^{s}(\Omega),
$$

with a suitable $s>0$, was considered in [3]. Then the imbedding

$$
W^{m, p}(\nu, \Omega) \smile W^{m, p_{1}}(\Omega)
$$


with $p_{1}=p s /(s+1)$ allowed us to reduce all considerations in [3] from the werghted Sobolev spaces to the nonuerghted ones, and consequently, to use the well-developped apparatus of the classical (i.e. nonweighted) Sobolev spaces (imbedding theorems etc.). Moreover, the assumption

$$
s>\frac{N}{p}
$$

onsuled the romparturs of the imbedding

$$
W^{m, p}(\nu, \Omega) \hookrightarrow W^{m-1 \cdot p}(\Omega) .
$$

Ilowever, the assumption (1.4) (together with (1.6)) is a "global" one in contrary to the assumptions (1.3). Hencr (1.4) is rather restrictive and excludes a big class of weight functions appearing in practical problems. Thelefore, it is the intention of this paper to remove the assumption (1.4) and to work cxclusively in we zghted Sobolev spaces, avoiding the "deviation" via classical Sobolev spaces expressed by the imbedding (1.5). The information available about the properties of weighted Sobolev spaces allow us to handle the problem derectly in weighted spaces (see e.g. OPIC' and KUFNER [18] for imbedding theorems in weighted Sobolev spaces).

Let us illustrate the advantage of our approach on the example mentioned already in [3]. The operator

$$
(A u)(x)=(-1)^{m} \sum_{|\alpha|=m} D^{\alpha}\left(\nu(x)\left|D^{\alpha} u(x)\right|^{p-2} D^{\alpha} u(x)\right)
$$

was considered there on $\Omega \subset \mathbb{R}^{2}$, where $\Omega$ is the square $\left\{\left(x_{1}, x_{2}\right) ;-1<x_{\imath}<\right.$ $<1,1=1,2\}$, with

$$
\nu(x)=\nu\left(x_{1}, x_{2}\right)= \begin{cases}1 & \text { for } x_{1} \leq 0 \\ x_{2}^{\lambda}\left(1-x_{1}\right)^{\gamma} & \text { for } x_{1}>0, x_{2}>0 \\ \left|x_{2}\right|^{\mu}\left(1-x_{1}\right)^{\gamma} & \text { for } x_{1}>0, x_{2}<0 .\end{cases}
$$

The operator $A$ was considered on the space $W^{m, p}(\nu, \Omega)$ from $(1.2)$ with $\nu_{\alpha}(x)=\nu(x)$ for $|\alpha|=m$. It follows from conditions (1.3) that it has to be

$$
\lambda, \mu \in]-1, p-1[
$$

while condition (1.4) (together with (1.6)) indicates that we have to choose

$$
\lambda, \mu, \gamma<\frac{p}{2}
$$

On the other hand if we use the approach described in this paper, we can avoid - at least for the formulation of the problem - the last restriction and consider more admissible values of the parameters $\lambda, \mu$ and $\gamma$.

The first attempt to remove assumption (1.4) and to derive the imbedding (1.7) directly, without introducing the intermediate space $W^{m, p_{1}}(\Omega)$ was made by KUFNER and LEONARDI [11], where also imbedding theorems for weighted Sobolev spaces have been used. In this paper we use weighted spaces systematically.

The paper is organized as follows. In Section 2 we define suitable function spaces and invite reader's attention to some useful imbedding theorems. In Section 3 we formulate various types of growth conditions on the coefficients of the differential operator $A$ from (1.1). Section 4 consists in formulation of the boundary value problem for the differential operator $A$ as an operator equation. We show in Section 5 , that it is possible to apply the degree theory in order to solve the corresponding operator equation. In Section 6 we present existence results and Section 7 contains concluding remarks and applications.

This paper, which generalizes the results of our preceding work [3], may be regarded as a continuation of the papers GUGLIELMINO and NICOLOSI [8], DRÁBEK and NICOLOSI $[4,5]$.

\section{Function spaces}

Let $m \in \mathbb{N}, p \in] 1, \infty\left[, \Omega\right.$ be an open subset of $\mathbb{R}^{N}(N \geq 1)$ and $\nu$ be a family of weight functions $\nu_{\alpha}=\nu_{\alpha}(x),|\alpha| \leq m$, i.e. every $\nu_{\alpha}$ is measurable and positive almost everywhere (a.e.) in $\Omega$. We will denote by

$$
W^{m, p}(\nu, \Omega)
$$

the set of all functions $u=u(x)$ defined a.e. in $\Omega$ such that their derivatives (in the sense of distributions) $D^{\alpha} u$ of order $|\alpha| \leq m$ belong to the weighted Lebesgue space $L^{p}\left(\nu_{\alpha}, \Omega\right)$, i.e. 


$$
\left\|D^{\alpha} u\right\|_{p, \nu_{\alpha}}=\left(\int_{\Omega}\left|D^{\alpha \alpha} u(x)\right|^{p} \nu_{\alpha}(x) d x\right)^{\frac{1}{p}}<\infty
$$

[Notice the difference between the space $W^{m, p}(\nu, \Omega)$ from $(2.1)$, where the weights $\nu_{\alpha}$ appear at all derivatives $D^{\alpha} u$, and the space $W^{m, p}(\nu, \Omega)$ from $(1.2)$, where they appear only at $D^{\alpha} u$ with $|\alpha|=m$.] If we suppose that

$$
\nu_{\alpha}^{-\frac{1}{p-1}} \in L_{l o c}^{1}(\Omega) \text { for }|\alpha| \leq m,
$$

then $W^{m, p}(\nu, \Omega)$ is a uniformly convex Banach space if equipped with the norm

$$
\|u\|_{m, p, \nu}=\left(\sum_{|\alpha| \leq m}\left\|D^{\alpha} u\right\|_{p, \nu_{\alpha}}^{p}\right)^{\frac{1}{p}}
$$

If we şuppose in addition, that

$$
\nu_{\alpha} \in L_{l o c}^{1}(\Omega) \text { for }|\alpha| \leq m
$$

then $C_{0}^{\prime \infty}(\Omega)$ is a subset of $W^{m, p}(\nu, \Omega)$ and we can define the space

$$
W_{0}^{m, p}(\nu, \Omega)
$$

as the closure of $C_{0}^{\infty}(\Omega)$ with respect to the norm (2.2). For details concerning the above assertions see e.g. NICOLOSI [17], GUGLIELMINO and NICOLOSI [7] or KUFNER and OPIC [12].

For the sake of brevity we will use the following notation. Given two Banach spaces $X, Y$ we will write $X \hookrightarrow Y$ or $X \hookrightarrow \hookrightarrow Y$ if $X \subseteq Y$ and the natural injection of $X$ into $Y$ is continuous or compact, respectively.

Similarly as in the case of classical Sobolev spaces, there are imbedding theorems for weighted Sobolev spaces of the type

$$
W^{1, p}(\nu, \Omega) \hookrightarrow L^{q}(\omega, \Omega)
$$

and

$$
W_{0}^{1, p}(\nu, \Omega) \hookrightarrow L^{q}(\omega, \Omega) .
$$

The imbeddings mentioned above follow from inequalities of the form

$$
\begin{aligned}
\left(\int_{\Omega}|u(x)|^{q} \omega(x) d x\right)^{\frac{1}{q}} & \leq C\left(\int_{\Omega}|u(x)|^{p} \nu_{0}(x) d x+\right. \\
& \left.+\sum_{i=1}^{N} \int_{\Omega}\left|\frac{\partial u}{\partial x_{i}}(x)\right|^{p} \nu_{i}(x) d x\right)^{\frac{1}{p}}
\end{aligned}
$$

and

$$
\left(\int_{\Omega}|u(x)|^{q} \omega(x) d x\right)^{\frac{1}{q}} \leq C\left(\sum_{i=1}^{N} \int_{\Omega}\left|\frac{\partial u}{\partial x_{\imath}}(x)\right|^{p} \nu_{\imath}(x) d x\right)^{\frac{1}{p}}
$$

Remark 2.1 For details concerning the conditions on $p, q, \omega, \nu_{0}, \nu_{1}, \ldots, \nu_{N}$ which guarantee the validity of (2.5) and (2.6), we refer to OPIC and KUFNER [10]. Let us emphasize that both possibilities $p>q$ and $p \leq q$ can occur in (2.3) and (2.4) and that in the case $p>q$ these imbeddings are simultaneously compact, while in the case $p \leq q$, some additional assumptions are needed.

Example 2.1 Let $\Omega$ be bounded, $\partial \Omega$ be locally lipschitzian, $\nu_{0}(x)=\nu_{1}(x)=\ldots=\nu_{N}(x)=(\operatorname{dist}(x, \partial \Omega))^{\lambda}, \omega(x$ $(\operatorname{dist}(x, \partial \Omega))^{\kappa}, \lambda, \kappa \in \mathbb{R}, \lambda<p-1$. Then (2.4) holds (and(2.6) holds for any $u \in C_{0}^{\infty}(\Omega)$ ) if and only if either

or

$$
1<p \leq q<\infty, \frac{N}{q}-\frac{N}{p}+1 \geq 0, \kappa \geq \lambda \frac{q}{p}-N+N \frac{q}{p}-q
$$

$$
1 \leq q<p<\infty, \kappa>\lambda \frac{q}{p}-1+\frac{q}{p}-q .
$$

Moreover, the imbedding (2.4) is compact if, for $p \leq q$, the last inequality in (2.7) is sharp (see OPIC and KUFNER [18], Theorem 21.5). 
Remark 2.2 We mentioned only imbeddings of $W^{1, p}(\nu, \Omega)$. Of course, by a repeated use of $(2.3)$ and (2.4) we can show that similar imbeddings hold also for $W^{m, p}(\nu, \Omega)$ and $W_{0}^{m, p}(\nu, \Omega)$, respectively, with $m>1$.

In the sequel we will use the following hypotheses.

Hypothesis 2.1 For $u \in W^{m, p}(\nu, \Omega)$ ( or $\left.u \in W_{0}^{m, p}(\nu, \Omega)\right)$, it is

$$
D^{\beta} u \in L^{q(\beta)}\left(\omega_{\beta}, \Omega\right) \text { for }|\beta|<m
$$

and

$$
\left\|D^{\beta} u\right\|_{q(\beta), \omega_{\beta}} \leq \tilde{c}_{\beta}\|u\|_{m, p, \nu}
$$

with $\tilde{c}_{\beta}$ independent of $u$, with some appropriate parameter $q(\beta) \geq 1$ and appropriate weight function $\omega_{\beta}$.

Remark 2.3 Similarly as in the case of classical Sobolev spaces, it is possible to derive also for weighted Sobolev spaces imbeddings into spaces of continuous or Hölder-continuous functions. For example, it can be shown that for $p>N$ the imbedding

$$
W^{1, p}(\nu, \Omega) \hookrightarrow C(\omega, \Omega)
$$

holds with an appropriate weight function $\omega$, where $C(\omega, \Omega)$ is the set of all continuous functions $u=u(x)$ on $\Omega$ for which

$$
\|u\|_{C(\omega, \Omega)}=\sup _{x \in \Omega}|\omega(x) u(x)|<\infty
$$

Example 2.2 Let $\Omega, \partial \Omega, \omega(x), \nu_{\imath}(x), i=0, \ldots, N$, be as in Example 2.1, $p>N$. Then the imbedding

$$
W_{0}^{1, p}(\nu, \Omega) \hookrightarrow C(\omega, \Omega)
$$

holds if and only if

$$
\kappa \geq \frac{\lambda}{p}-1+\frac{N}{p}
$$

provided $\lambda \neq p-1$ (see BROWN and OPIC [2], where also the questions of compactness of imbeddings of the form (2.10) and imbeddings into spaces of Hölder-continuous functions are dealt with).

\section{Growth conditions}

The weighted Sobolev space $W^{m, p}(\nu, \Omega)$ (with $\nu=\left\{\nu_{\alpha} ;|\alpha| \leq m\right\}$ ) in which we are looking for the weak solution is closely related to the growth conditions on the "coefficients" $A_{\alpha}(x, \xi)$ which appear in the definition of the differential operator $A$ in (1.1).

Let $k=k(m, N)$ be the number of all (different) multiindices $\alpha=\left(\alpha_{1}, \alpha_{2}, \ldots, \alpha_{N}\right)$ (i.e. $N$-dimensional vectors whose components $\alpha_{1}$ are nonnegative integers) of the length $|\alpha|=\alpha_{1}+\alpha_{2}+\ldots+\alpha_{N}$ not exceeding $m$. (In other words, $k$ is the number of all derivatives

$$
D^{\alpha} u(x)=\frac{\partial^{|\alpha|} u(x)}{\partial x_{1}^{\alpha_{1}} \partial x_{2}^{\alpha_{2}} \ldots \partial x_{N}^{\alpha_{N}}}
$$

$|\alpha| \leq m$.) Assume that the function $A_{\alpha}(x, \xi), x \in \Omega, \xi \in \mathbf{R}^{k}, \xi=\left\{\xi_{\beta} ;|\beta| \leq m\right\}$, satisfies the Carathéodory condition, i.e. it is continuous in $\xi$ for a.e. $x \in \Omega$ and measurable in $x$ for every $\xi \in \mathbf{R}^{k}$.

Definition 3.1 For $p>1$ denote by $p^{\prime}=\frac{p}{p-1}$ the exponent conjugate to $p$. Let $g_{\alpha} \in L^{p^{\prime}}(\Omega)$ and $c_{\alpha} \geq 0$ be a constant. We say that the coefficients $A_{\alpha}$ of the differential operator $A$ from (1.1) statisfy the growth conditions of type $(A)$ if the estimate

$$
\left|A_{\alpha}(x, \xi)\right| \leq \nu_{\alpha}^{\frac{1}{p}}(x)\left[g_{\alpha}(x)+c_{\alpha} \sum_{|\beta| \leq m} \nu_{\beta}^{\frac{1}{p^{\prime}}}(x)\left|\xi_{\beta}\right|^{p-1}\right]
$$

holds for a.e. $x \in \Omega$ and every $\xi \in \mathbf{R}^{k} ;|\alpha| \leq m$.

Definition 3.2 Let $q(\beta)$ and $\omega_{\beta},|\beta| \leq m-1$, be the parameters appearing in Hypothesis 2.1 , and let $g_{\alpha}$ and $c_{\alpha}$ be as in the foregoing Definition 3.1. We say that the coefficients $A_{\alpha}$ satisfy the growth conditions 
of type $(B)$ if the estimate

$$
\begin{aligned}
\left|A_{\alpha v}(x, \xi)\right| \leq \nu_{\alpha}^{\frac{1}{p}}(x)\left[g_{\alpha}(x)\right. & +c_{\alpha} \sum_{|\beta|=m} \nu_{\beta \beta}^{\frac{1}{p^{\prime}}}(x)\left|\xi_{\beta}\right|^{p-1}+ \\
& \left.+c_{\alpha} \sum_{|\beta| \leq m-1} \omega_{\beta}^{\frac{1}{p^{\prime}}}(x)\left|\xi_{\beta}\right|^{\frac{q(\beta)}{p^{\prime}}}\right]
\end{aligned}
$$

holds for a.e. $x \in \Omega$ and every $\xi \in \mathbf{R}^{k} ;|\alpha| \leq m$.

Definition 3.3 Let $g_{\alpha}, c_{\alpha}, q(\beta)$ and $\omega_{13}$ be as in the foregoing Definition 3.2. Moreover, let $\check{g}_{\alpha} \in L^{q(\alpha)^{\prime}}$ for $|\alpha| \leq m-1, q(\alpha)^{\prime}=q(\alpha) /(q(\alpha)-1)$. We say that the coefficients $A_{\alpha}$ satisfy the growth conditions of type ((') if the following estimates hold for a.e. $x \in \Omega$ and every $\xi \in \mathbb{R}^{k}$ :

(i) For $|\alpha|=m$,

$$
\begin{aligned}
\left|A_{\alpha}(x, \xi)\right| \leq \nu_{\alpha}^{\frac{1}{p}}(x)\left[g_{\alpha}(x)\right. & +c_{\alpha} \sum_{|\beta|=m} \nu_{\beta}^{\frac{1}{p^{p}}}\left|\xi_{\beta}\right|^{p-1}+ \\
& \left.+c_{\alpha} \sum_{|\beta| \leq m-1} \omega_{\beta}^{\frac{1}{p^{\prime}}}(x)\left|\xi_{\beta}\right|^{\frac{q(\beta)}{p^{\prime}}}\right]
\end{aligned}
$$

(ii) for $|\alpha| \leq m-1$,

$$
\begin{aligned}
\left|A_{\alpha}(x, \xi)\right| \leq \omega_{\alpha}^{\frac{1}{q(\alpha)}}(x)\left[\tilde{g}_{\alpha}(x)\right. & +c_{\alpha} \sum_{|\beta|=m} \nu_{\beta}^{\frac{1}{q(\alpha)}}(x)\left|\xi_{\beta}\right|^{\frac{p}{q(\alpha)^{\prime}}}+ \\
& \left.+c_{\alpha} \sum_{|\beta| \leq m-1} \omega_{\beta}^{\frac{1}{q(\alpha)^{\prime}}}(x)\left|\xi_{\beta}\right|^{\frac{q(\beta)}{q(\alpha)^{\prime}}}\right] .
\end{aligned}
$$

Let us denote

$$
\kappa_{1}=m-\frac{N}{p}
$$

and suppose that $\kappa_{1}>0$, i.e. $m p>N$. Due to Remark 2.3, we can - besides (2.8) - consider also imbeddings of $W^{m, p}(\nu, \Omega)$ into spaces $C^{\prime}(\omega, \Omega)$, i.e. for $u \in W^{m, p}(\nu, \Omega)$ (or for $u \in W_{0}^{m, p}(\nu, \Omega)$ ) it is

$$
D^{\beta} u \in C\left(\omega_{\beta}, \Omega\right)
$$

for $|\beta|<\kappa_{1}$ and

$$
\sup _{x \in \Omega}\left|\omega_{\beta}(x) D^{\beta} u(x)\right| \leq \tilde{c}_{\beta}\|u\|_{m, p, \nu}
$$

for $|\beta|<\kappa_{1}$, with $\tilde{c}_{\beta}>0$ independent of $u \in W^{m, p}(\nu, \Omega)$. Taking into account also these imbeddings, we can modify our foregoing growth conditions of type $(A)-(C)$. For this purpose, let us write $\xi \in \mathbb{R}^{k}$ in the form $\xi=(\kappa, \tilde{\kappa})$, where $\kappa=\left\{\xi_{\beta} ;|\beta|<\kappa_{1}\right\}$, and denote

$$
h(x, \kappa)=\sum_{|\beta|<\kappa_{1}}\left|\omega_{\beta}(x) \xi_{\beta}\right| .
$$

If we denote by $h(x, u(x))$ the expression

$$
\sum_{|\beta|<\kappa_{1}}\left|\omega_{\beta}(x) D^{\beta} u(x)\right|
$$

then it is, in view of (3.7),

$$
|h(x, u)| \leq c\|u\|_{m, p, \nu}
$$

for $u \in W^{m, p}(\nu, \Omega)$.

Definition 3.4 Let us use the same notation as in the preceding definitions. Suppose, moreover, that $\hat{g}_{\alpha} \in L^{1}(\Omega)$ for $|\alpha|<\kappa_{1}$. We say that the coefficients $A_{\alpha}$ satisfy the growth conditions of type $(D)$, if there is a positive, continuous, nondecreasing function $G(t), t \geq 0$, such that the following estimates hold for a.e. $x \in \Omega$ and every $\xi \in \mathbb{R}^{k}$ :

(i) For $|\alpha|=m$, 


$$
\begin{aligned}
\left|A_{\alpha}(x, \xi)\right| \leq\left(i ( h ( x , \kappa ) ) \nu _ { \alpha } ^ { \frac { 1 } { p } } ( x ) \left[g_{\alpha}(x)\right.\right. & +c_{\alpha} \sum_{|\beta|=m} \nu_{\beta}^{\frac{1}{p^{\prime}}}(x)\left|\xi_{\beta}\right|^{p-1}+ \\
& \left.+c_{\alpha} \sum_{\kappa_{1} \leq|\beta| \leq m-1} \omega_{\beta}^{\frac{1}{p^{p}}}(x)\left|\xi_{\beta}\right|^{\frac{q(\beta)}{p^{\prime}}}\right] ;
\end{aligned}
$$

(ii) for $\kappa_{1} \leq|\alpha| \leq m-1$,

$$
\begin{aligned}
& \left|A_{\alpha}(x, \xi)\right| \leq\left(i ( h ( x , \kappa ) ) \omega _ { \alpha } ^ { \frac { 1 } { q ( \alpha ) } } ( x ) \left[\tilde{g}_{\alpha}(x)+c_{\alpha} \sum_{|\beta|=m} \nu_{\beta}^{\frac{1}{q(\alpha)^{\prime}}}(x)\left|\xi_{\beta}\right|^{\frac{p}{q(\alpha)^{\prime}}}+\right.\right. \\
& \left.+c_{\alpha} \sum_{\kappa_{1} \leq|\beta| \leq m-1} \omega_{\beta}^{\frac{1}{q(\alpha)^{\prime}}}\left|\xi_{\beta}\right|^{\frac{q(\beta)}{q(\alpha)^{\prime}}}\right]
\end{aligned}
$$

(iii) for $|\alpha|<\kappa_{1}$,

$$
\begin{aligned}
\left|A_{\alpha}(x, \xi)\right| \leq G(h(x, \kappa)) \omega_{\alpha}(x)\left[\hat{g}_{\alpha}(x)\right. & +c_{\alpha} \sum_{|\beta|=m} \nu_{\beta}(x)\left|\xi_{\beta}\right|^{p}+ \\
& \left.+c_{\alpha} \sum_{\kappa_{1} \leq|\beta| \leq m-1} \omega_{\beta}(x)\left|\xi_{\beta}\right|^{q(\beta)}\right] .
\end{aligned}
$$

Remark 3.1 It is easy to show that the growth conditions of type (D) contain the previous ones as special cases (e.g., we set $G(t) \equiv 1$ if $\kappa_{1} \leq 0$ and, consequently, the set of $\xi_{\beta}$ with $|\beta|<\kappa_{1}$ is empty). Therefore, we can concentrate on Definition 3.4 .

\section{Operator representation}

Let us consider a boundary value problem for the partial differential equation

$$
A u=f \text { in } \Omega,
$$

where $A$ is the differential operator of the form (1.1). Let $V$ be a closed vector subspace of the weighted Sobolev space $W^{m, p}(\nu, \Omega)$ from (2.1) such that

$$
W_{0}^{m, p}(\nu, \Omega) \subseteq V \subseteq W^{m, p}(\nu, \Omega) .
$$

The choice of $V$ depends on the boundary conditions, which appear in our boundary value problem. Roughly speaking, $V$ consists of all functions from $W^{m, p}(\nu, \Omega)$ which "satisfy the homogeneous boundary conditions" - it is $V=W_{0}^{m, p}(\nu, \Omega)$ if we consider the Dirichlet problem for the equation (4.1), and it is $V=W^{m, p}(\nu, \Omega)$ if we consider the Neumann problem.

For $j \in \mathbf{N}$ we shall write

$$
D^{\jmath} u=\left\{D^{\beta} u ;|\beta|=j\right\} .
$$

Let

$$
a(u, \varphi)=\sum_{|\alpha| \leq m} \int_{\Omega} A_{\alpha}\left(x, u(x), \ldots, D^{m} u(x)\right) D^{\alpha} \varphi(x) d x
$$

be the form (linear in $\varphi$ ) associated with the differential operator $A$.

Definition 4.1 Let $u_{0} \in W^{m, p}(\nu, \Omega)$ be a fixed function. $A$ function $u=u_{0}+v, v \in V$, satisfying the integral identity

$$
a\left(u_{0}+v, \varphi\right)=\int_{\Omega} f(x) \varphi(x) d x
$$

for every $\varphi \in V$, will be called the weak solution of the nonhomogeneous boundary value problem for the equation (4.1)

Remark 4.1 In order to avoid tedious calculations with expressions invol-

ving the terms $u_{0}+v, D^{\alpha}\left(u_{0}+v\right)$, etc., we will suppose in the sequel that $u_{0} \equiv 0$, i.e. instead of (4.4) we will be looking for the weak solution of the homogeneous boundary value problem, i.e. for the function 
$u \in V$ satisfying the integral identity

$$
a(u, \varphi)=\int_{\Omega} f(x) \varphi(x) d . r
$$

for evely $\varphi \in V$. The reader should bear in mind that the simple shift $u \rightarrow u_{0}+u$ transfers existence results for the homogeneous problem to existence results for the nonhomogeneous one.

Lemma 4.1 Let the funrtions $A_{\alpha}(x, \xi)$ satisfy the growth conditions of type $(A),(B),\left(C^{*}\right)$ or $(D)$ (se' De fintions 3.1-3.4). Then the form $a(u, \varphi)$ from $(4.3)$ is well-defined for any $u, \varphi \in W^{m, p}(\nu, \Omega)$. Moreover, the rex cxsts a continuous postive function $H(t)$ defined in $[0, \infty[$ such that

$$
|a(u, \varphi)| \leq H\left(\|u\|_{m, p, \nu}\right)\|\varphi\|_{m, p, \nu}
$$

Proof It is sufficient to consider the growth conditions of type $(D)$ (see Remark 3.1 ). Then it follows from (3.10) (for $|\alpha|=m$ ) that

$$
\begin{aligned}
& \left|A_{\alpha}\left(x, u(x), \ldots, D^{m} u(x)\right)\right| \leq G(h(x, u(x))) \nu_{\alpha}^{\frac{1}{p}}(x) . \\
& \cdot\left[\left|g_{\alpha}(x)\right|+c_{\alpha} \sum_{|\beta|=m} \nu_{\beta}^{\frac{1}{p^{\prime}}}(x)\left|D^{\beta} u(x)\right|^{p-1}+\right. \\
& \left.+c_{\alpha} \sum_{\kappa_{1} \leq|\beta| \leq m-1} \omega_{\beta}^{\frac{1}{p^{\prime}}}(x)\left|D^{\beta} u(x)\right|^{\frac{q(\beta)}{p^{\prime}}}\right]
\end{aligned}
$$

and consequently

$$
\begin{aligned}
& \int_{\Omega}\left|A_{\alpha}\left(x, u(x), \ldots, D^{m} u(x)\right) D^{\alpha} \varphi(x)\right| d x \leq \\
& \leq \int_{\Omega} G(h(x, u(x))) \nu_{\alpha}^{\frac{1}{p}}(x)\left|D^{\alpha} \varphi(x)\right|\left|g_{\alpha}(x)\right| d x+ \\
& +c_{\alpha} \sum_{|\beta|=m} \int_{\Omega} G(h(x, u(x))) \nu_{\alpha}^{\frac{1}{p}}(x)\left|D^{\alpha} \varphi(x)\right| \nu_{\beta}^{\frac{1}{p^{\prime}}}(x)\left|D^{\beta} u(x)\right|^{\frac{p}{p^{p}}} d x+ \\
& +c_{\alpha} \sum_{\kappa_{1} \leq|\beta| \leq m-1} \int_{\Omega} G(h(x, u(x))) \nu_{\alpha}^{\frac{1}{p}}(x)\left|D^{\alpha} \varphi(x)\right| \omega_{\beta}^{\frac{1}{p^{\prime}}}(x)\left|D^{\beta} u(x)\right|^{\frac{q(\beta)}{p^{\prime}}} d x .
\end{aligned}
$$

It follows from the monotonicity of the function $G$ and from (3.9) that

$$
G(h(x, u(x))) \leq G\left(c\|u\|_{m, p, \nu}\right)
$$

We will estimate the integrals in (4.7) using (4.8) and the Hölder inequality with the exponents $p$ and $p^{\prime}$ :

$$
\begin{aligned}
& \int_{\Omega}\left|A_{\alpha}\left(x, u(x), \ldots, D^{m} u(x)\right) D^{\alpha} \varphi(x)\right| d x \leq \\
& \leq G\left(c\|u\|_{m, p, \nu}\right)\left\|D^{\alpha} \varphi\right\|_{p, \nu_{\alpha}}\left[\left\|g_{\alpha}\right\|_{p^{\prime}}+\right. \\
& \left.+c_{\alpha} \sum_{|\beta|=m}\left\|D^{\beta} u\right\|_{p, \nu_{\beta}}^{p-1}+c_{\alpha} \sum_{\kappa_{1} \leq|\beta| \leq m-1}\left\|D^{\beta} u\right\|_{q(\beta), \omega_{\beta}}^{\frac{q(\beta)}{p^{\prime}}}\right] .
\end{aligned}
$$

Since we have $\left\|D^{\beta} u\right\|_{p, \nu_{\beta}} \leq\|u\|_{m, p, \nu}$ by definition of the norm in $W^{m, p}(\nu, \Omega)$ and $\left\|D^{\beta} u\right\|_{q(\beta), \omega_{\beta}} \leq \tilde{\boldsymbol{c}}_{\beta}\|u\|_{m, p, \nu}$ due to Hypothesis 2.1, we obtain from (4.9)

$$
\begin{aligned}
& \int_{\Omega}\left|A_{\alpha}\left(x, u(x), \ldots, D^{m} u(x)\right) D^{\alpha} \varphi(x)\right| d x \leq \\
& \leq\left\|D^{\alpha} \varphi\right\|_{p, \nu_{\alpha}} H_{\alpha}\left(\|u\|_{m, p, \nu}\right) \leq\|\varphi\|_{m, p, \nu} H_{\alpha}\left(\|u\|_{m, p, \nu}\right)
\end{aligned}
$$

for $|\alpha|=m$, where $H_{\alpha}(t)$ is some positive continuous function. 
A similar estimate can be derived by analogous arguments for $\kappa_{1} \leq|\alpha| \leq m-1$ from the condition (3.11) (notice that we use the Hölder inequality with the exponents $\left.q(\alpha), q(\alpha)^{\prime}\right)$.

Finally, for $|\alpha|<\kappa_{1}$, we use the condition (3.12) which leads to the estimate

$$
\begin{aligned}
& \int_{\Omega}\left|A_{\alpha}\left(x, u(x), \ldots, D^{m} u(x)\right) D^{\alpha} \varphi(x)\right| d x \leq \\
& \leq \int_{\Omega}\left(i(h(x, u(x))) \omega_{\alpha}(x)\left|D^{\alpha} \varphi(x)\right| \hat{g}_{\alpha}(x) \mid d x+\right. \\
& +c_{\alpha \alpha} \sum_{|\beta|=m} \int_{\Omega}\left(i(h(x, u(x))) \omega_{\alpha}(x)\left|D^{\alpha} \varphi(x)\right| \nu_{\beta}(x)\left|D^{\beta} u(x)\right|^{p} d x+\right. \\
& +c_{\alpha} \sum_{\kappa_{1} \leq \beta \leq m-1} \int_{\Omega}\left(i(h(x, u(x))) \omega_{\alpha}(x)\left|D^{\alpha} \varphi(x)\right| \omega_{\beta}(x)\left|D^{\beta} u(x)\right|^{q(\beta)} d x .\right.
\end{aligned}
$$

Since $D^{\alpha} \varphi \in C^{\prime}\left(\omega_{\alpha}, \Omega\right)$ for $|\alpha|<\kappa_{1}$ (see (3.6)), we obtain from (4.11), (3.6)

$$
\begin{aligned}
& \int_{\Omega}\left|A_{\alpha}\left(x, u(x), \ldots, D^{m} u(x)\right) D^{\alpha} \varphi(x)\right| d x \leq \\
& \leq G\left(c\|u\|_{m, p, \nu}\right) \cdot \sup _{x \in \Omega}\left|\omega_{\alpha}(x) D^{\alpha} \varphi(x)\right|\left[\left\|\hat{g}_{\alpha}\right\|_{1}+\right. \\
& \left.+c_{\alpha} \sum_{|\beta|=m}\left\|D^{\beta} u\right\|_{p, \nu_{\beta}}^{p}+c_{\alpha} \sum_{\kappa_{1} \leq|\beta| \leq m-1}\left\|D^{\beta} u\right\|_{q(\beta), \omega_{\beta}}^{q(\beta)}\right] .
\end{aligned}
$$

Now, using the fact that $\sup _{x \in \Omega}\left|\omega_{\alpha}(x) D^{\alpha} \varphi(x)\right| \leq \tilde{c}_{\alpha}\|\varphi\|_{m, p, \nu}$ for $|\alpha|<\kappa_{1}$ (see(3.7)), we obtain from (4.12) an estimate of the form (4.10), but for $|\alpha|<\kappa_{1}$.

Since

$$
|a(u, \varphi)| \leq \sum_{|\alpha| \leq m} \int_{\Omega}\left|A_{\alpha}\left(x, u(x), \ldots, D^{m} u(x)\right) D^{\alpha} \varphi(x)\right| d x,
$$

we finally obtain the estimate (4.6) taking

$$
H(t)=\sum_{|\alpha| \leq m} H_{\alpha}(t) .
$$

Since $a(u, \varphi)$ is linear with respect to $\varphi$, the expression $a(u, \cdot)$ represents the value of a continuous linear functional $F \in V^{*}$ (the dual space):

$$
a(u, \varphi)=\langle F, \varphi\rangle
$$

(here $\langle\cdot, \cdot\rangle$ denotes the duality between $V^{*}$ and $V$ ). It follows from Lemma 4.1 that

$$
\|F\|_{V *} \leq H\left(\|u\|_{V}\right)
$$

The dependence of $F$ on $u$ (cf.(4.13)) will be expressed by writing

$$
F=T u \text {. }
$$

Hence we defined the operator $T: V \rightarrow V^{*}$ by the relation

$$
a(u, \varphi)=\langle T u, \varphi\rangle, u, \varphi \in V
$$

It follows from (4.14) that

$$
\|T u\|_{V *} \leq H\left(\|u\|_{V}\right)
$$

which means that the operator $T$ is bounded (i.e. $T$ maps bounded sets in $V$ onto bounded sets in $V^{*}$ ).

Using the properties of the Nermytskii operator acting between weighted spaces we are able to prove also the continuity of $T$. 
Proposition 4.1 Let the functions.s $A_{\alpha}=A_{\alpha}(x, \xi)$ dcfined on $\Omega \times \mathbf{R}^{k}$ satisfy the ('arathrodory condations and one of the grouth conditions of the type $(A),(B),\left(C^{\prime}\right)$ or $(D)$. Then the operator $T: V \rightarrow V^{*}$ defined by (4.15) is bounded and continuous.

The proof follows the ideas of the proof of Theorem 3.5 in [3]. Let us only emphasize that due to our assumptions we can identify the space $V$ with a closed subspace of the product space

$$
P=\prod_{|\beta| \leq m} X_{\beta}
$$

where

$$
\begin{cases}X_{\beta}=L^{p}\left(\nu_{\beta}, \Omega\right) & \text { for }|\beta|=m, \\ X_{\beta}=L^{q(\beta)}\left(\omega_{\beta}, \Omega\right) & \text { for } \kappa_{1} \leq|\beta| \leq m-1, \\ X_{\beta}=C^{\prime}\left(\omega_{\beta}, \Omega\right) & \text { for }|\beta|<\kappa_{1} .\end{cases}
$$

Note that $\kappa_{1}=m-\frac{N}{p}$ and if $\kappa_{1} \leq 0$ then we use only the first two spaces in (4.17). Let us also recall that the norm in $C^{\prime}\left(\omega_{\beta}, \Omega\right)$ is defined by $(2.11)$ and in fact it is the $L^{\infty}$-norm of $\omega_{\beta} u$.

Remark 4.2 According to the definition of the operator $T$ (see (4.15)) a function $u \in V$ is a weak solution of our homogeneous boundary value problem for the differential equation (4.1) if the identity

$$
\langle T u, \varphi\rangle=\int_{\Omega} f(x) \varphi(x) d x
$$

holds for every $\varphi \in V$. Moreover, we can suppose that $f \in V^{*}$, replace the integral on the right-hand side of (4.18) by $\langle f, \varphi\rangle$ and look for $u \in V$ satisfying

$$
\langle T u, \varphi\rangle=\langle f, \varphi\rangle
$$

for any $\varphi \in V$. Since (4.19) can be rewritten as the operator equation

$$
T u=f
$$

for our operator $T: V \rightarrow V^{*}$, we thus obtain a more general setting of our problem: "Find $v \in V$ such that (4.19) holds for any $\varphi \in V$."

Remark 4.3 To be more specific, we will suppose that the right-hand side $f$ in $(4.1)$ is of the form

$$
f=\sum_{|\alpha| \leq m}(-1)^{|\alpha|} D^{\alpha} f_{\alpha},
$$

where the family $\left\{f_{\alpha} ;|\alpha| \leq m\right\}$ satisfies the following conditions

$$
\begin{cases}f_{\alpha} \in\left(L^{p}\left(\nu_{\alpha}, \Omega\right)\right)^{*}=L^{p^{\prime}}\left(\nu_{\alpha}^{1-p^{\prime}}, \Omega\right) & \text { for }|\alpha|=m \\ f_{\alpha} \in\left(L^{q(\alpha)}\left(\omega_{\alpha}, \Omega\right)\right)^{*}=L^{q(\alpha)^{\prime}}\left(\omega_{\alpha}^{1-q(\alpha)^{\prime}}, \Omega\right) & \text { for } \kappa_{1} \leq|\alpha| \leq m-1 \\ f_{\alpha} \in L^{1}\left(\omega_{\alpha}^{-1}, \Omega\right) \subset\left(L^{\infty}\left(\omega_{\alpha}, \Omega\right)\right)^{*} & \text { for }|\alpha|<\kappa_{1}\end{cases}
$$

Then the operator equation (4.20) means that the integral identity

$$
\begin{array}{r}
\sum_{|\alpha| \leq m} \int_{\Omega} A_{\alpha}\left(x, u(x), \ldots, D^{m} u(x)\right) D^{\alpha} \varphi(x) d x= \\
=\sum_{|\alpha| \leq m} \int_{\Omega} f_{\alpha}(x) D^{\alpha} \varphi(x) d x
\end{array}
$$

holds for every $\varphi \in V$.

\section{Degree of the mapping}

Our existence results are based on the solvability of the operator equation (4.20). The main tool for proving the existence of the solution of $(4.20)$ will be the degree theory for generalized monotone mappings developed by BROWDER [1] and SKRYPNIK [19]. However, in order to apply this theory, we need to prove that the operator $T: V \rightarrow V^{*}$ satisfies the so called condition $\alpha(V)$ (sometimes also called condition $\left.(S)_{+}\right)$. 
Definition 5.1 The operator $T: V \rightarrow V^{*}$ is said to satisfy rondition $\alpha(V)$ if the assumptions

$$
u_{n}-u_{0}(\text { weakly) in } V \text { for } n \rightarrow \infty \text {, }
$$

$$
\limsup _{n \rightarrow \infty}\left\langle T u_{n}, u_{n}-u_{0}\right\rangle \leq 0
$$

imply the strong convergence $u_{n} \rightarrow u_{0}$ in $V$.

We will need several hypotheses.

Hypothesis 5.1 The compact imbedding

$$
V \hookrightarrow \hookrightarrow W^{m-1, p}(\nu, \Omega)
$$

holds.

Remark 5.1 Note that the symbol $\nu$ means $\left\{\nu_{\alpha} ;|\alpha| \leq m\right\}$ in the space on the left-hand side in (5.3) and $\left\{\nu_{\alpha} ;|\alpha| \leq m-1\right\}$ in the space on the right-hand side in (5.3). The imbedding (5.3) is a natural one. It holds in the case of classical Sobolev spaces (i.e. if $\nu_{\alpha}(x) \equiv 1,|\alpha| \leq m$ ) if $\Omega$ is bounded and its boundary $\partial \Omega$ is lipschitzian.

Let, us denote

$$
H^{m-1, q}(\omega, \Omega)=\left\{u ; D^{\beta} u \in X_{\beta},|\beta| \leq m-1\right\},
$$

where $X_{\beta}$ are defined in (4.17).

Hypothesis 5.2 The compact imbedding

$$
V \hookrightarrow \hookrightarrow H^{m-1, q}(\omega, \Omega)
$$

holds.

In further hypotheses, we shall write the basic vector $\xi=\left\{\xi_{\beta} ;|\beta| \leq m\right\} \in \mathbf{R}^{k}$ in the form

$$
\xi=(\kappa, \eta, \zeta),
$$

where

$$
\begin{aligned}
& \zeta=\left\{\zeta_{\beta} ;|\beta|=m\right\} \in \mathbf{R}^{k_{1}} \text { with } \zeta_{\beta}=\xi_{\beta}, \\
& \eta=\left\{\eta_{\beta} ; \kappa_{1} \leq|\beta| \leq m-1\right\} \in \mathbf{R}^{k_{2}} \text { with } \eta_{\beta}=\xi_{\beta}, \\
& \kappa=\left\{\kappa_{\beta} ;|\beta|<\kappa_{\}} \in \mathbb{R}^{k_{3}} \text { with } \kappa_{\beta}=\xi_{\beta} .\right.
\end{aligned}
$$

If $\kappa_{1}=m-\frac{N}{p} \leq 0$, we shall write simply

$$
\xi=(\eta, \zeta)
$$

and the same notation will be used if it will be unnecessary to distinguish $\xi_{\beta}$ with $|\beta|<\kappa_{1}$ and $\xi_{\beta}$ with $|\beta| \in\left[\kappa_{1}, m-1\right]$.

Hypothesis 5.3 Let $G_{1}$ be a continuous, positive, nonincreasing function on $\left[0, \infty\left[, G_{2}\right.\right.$ a continuous, positive, nondecreasing function on $\left[0, \infty\left[\right.\right.$. We shall suppose that for every $\xi=(\kappa, \eta, \zeta) \in \mathbb{R}^{k}$ and for a.e. $x \in \Omega$, the following ellipticity condition holds:

$$
\begin{gathered}
\sum_{|\alpha|=m} A_{\alpha}(x, \kappa, \eta, \zeta) \zeta_{\alpha} \geq G_{1}(h(x, \kappa)) \sum_{|\beta|=m} \nu_{\beta}(x)\left|\zeta_{\beta}\right|^{p}- \\
-G_{2}(h(x, \kappa)) \sum_{\kappa_{1} \leq|\beta| \leq m-1} \omega_{\beta}(x)\left|\eta_{\beta}\right|^{q(\beta)} .
\end{gathered}
$$

where $h(x, \kappa)$ is defined by (3.8). If $\kappa_{1}<0$, we put $G_{1}(t)=G_{2}(t) \equiv c$ with c a positive constant.

Hypothesis 5.4 We shall suppose that the differential operator A from (2.1) is monotone in its principal part, i.e. for every $(\eta, \zeta) \in \mathbb{R}^{k},(\eta, \hat{\zeta}) \in \mathbf{R}^{k}$ with $\zeta \neq \hat{\zeta}$, and for a.e. $x \in \Omega$, the following monotonicity condition holds:

$$
\left[A_{\alpha}(x ; \eta, \zeta)-A_{\alpha}(x ; \eta, \hat{\zeta})\right]\left(\zeta_{\alpha}-\hat{\zeta}_{\alpha}\right)>0
$$

Hypothesis 5.5 The set $\Omega$ has a finite Lebesgue measure. 
Proposition 5.1 Let IIypothese's 5.1 - 5.5 br satusficd and let the corfficents $A_{\alpha}=A_{\alpha}(x, \xi)$ satısfy the growth conditions of type $(D)$. Then the opcrator $T$ defined by (4.15) satisfies condition $\alpha(V)$.

The proof follows the ideas of the proof of Lemma 4.4 in [3].

Remark 5.2 The properties of the mapping $T: V \rightarrow V^{*}$ just derived allow us to introduce its degree

$$
\operatorname{Deg}\left[T ; B_{R}, 0\right]
$$

with respect to the ball $B_{R}=\left\{u \in V ;\|u\|_{m, p, \nu} \leq R\right\}$ and the point $0 \in V^{*}$.

For details concerning the degree (5.7) see SKRYPNIK [19]. In the foregoing steps we have shown that $\operatorname{Deg}\left[T ; B_{R}, 0\right]$ is well-defined and has the properties stated in [19].

\section{Existence results}

In this section we present several existence results concerning the weak solvability of homogeneous boundary value problem for the equation (4.1). Recall that the right hand side $f$ is assumed to be of the form (4.21). Recall also that the results of this section can be easily extended to the case of nonhomogeneous boundary value problem (cf. Remark 4.1).

Let us point out that we dealt only with the most general growth conditions of type (D) (see Definition 3.4). However, existence results hold also (with obvious modifications) for the weaker growth conditions - - compare with Remark 3.1. For the simplest growth conditions of type (A), the existence of a (weak) solution was proved (for the Dirichlet problem) in KUFNER and OPIC [13] (see also KUFNER and SÄNDIG [14], Section 17).

Note also that $\Omega$ may be possibly an unbounded domain in $\mathbf{R}^{N}$. However, we have to assume that meas $\Omega<\infty$.

Theorem 6.1 Let $T: V \rightarrow V^{*}$ be defined by (4.15), the coefficients $A_{\alpha}(x, \xi)$ satisfy the qrowth condition.s of type (D) and the right-hand side $f$ is of the form (4.21). Let us assume that Hypotheses $5.1-5.5$ are satisfied and, moreover, assume that there exists a number $R>0$ such that

$$
\sum_{|\alpha| \leq m} \int_{\Omega}\left[A_{\alpha}\left(x, u(x), \ldots, D^{m} u(x)\right)-f_{\alpha}(x)\right] D^{\alpha} u(x) d x \geq 0
$$

holds for every $u \in V,\|u\|_{m, p, \nu}=R$.

Then the boundary value problem for the differential equation (4.1) has at least one weak solution $u \in V$ such that $\|u\|_{m, p, \nu} \leq R$.

Proof Let us introduce the operator $T_{f}: V \rightarrow V^{*}$ by

$$
T_{f} u=T u-f .
$$

Then the condition (6.1) can be written as

$$
\left\langle T_{f} u, u\right\rangle \geq 0 .
$$

If there is $u \in V$ with $\|u\|_{m, p, \nu}=R$ such that $T_{f} u=0$, then $T u=f$ and our assertion is proved (cf. Remark 4.3).

If there is no such $u \in V$ then condition (6.2) and Theorem 1.3.4 in SKRYPNIK [19] imply that the degree of $T_{f}$ satisfies

$$
\operatorname{Deg}\left[T_{f} ; B_{R}, 0\right]=1 \text {. }
$$

But then it follows from the basic property of the degree that there exists at least one $u \in B_{R}$ such that $T_{f} u=0$, i.e.

$$
T u=f
$$

Remark 6.1 A useful tool in the Leray-Lions theory of monotone operators is the concept of coercivity. An operator $T: V \rightarrow V^{*}$ is called coercive on $V$ if 


$$
\lim _{\|u\|_{V \rightarrow \infty}} \frac{\langle T u, u\rangle}{\|u\|_{V}}=\infty
$$

Obviously, this condition can help us to overcome the difficulties arising if we would like to verify the condition (6.1). Therefore, let us introduce an additional assumption.

Hypothesis 6.1 Suppose that there are positive constants $c_{1}, c_{2}, c_{3}$ such that the following coercivity condition holds for all $\xi \in \mathbb{R}^{k}$ and a.e. $x \in \Omega$ :

$$
\begin{aligned}
& \sum_{|\alpha| \leq m} A_{\alpha}(x, \xi) \xi_{\alpha} \geq c_{1} \sum_{|\alpha|=m} \nu_{\alpha}(x)\left|\xi_{\alpha}\right|^{p}+ \\
& +c_{2} \nu_{\theta}(x)\left|\xi_{\Theta}\right|^{p}-c_{3},
\end{aligned}
$$

where $\Theta$ is the zero multiinde $x(0,0, \ldots, 0)$.

Lemma 6.1 Let Hypotheses 5.1 and 6.1 be fulfilled. Then the operator $T: V \rightarrow V^{*}$ defined by (4.15) satisfies (6.3).

Proof It follows from Hypothesis 5.1 that the following imbeddings hold:

$$
V \hookrightarrow \hookrightarrow W^{m-1, p}(\nu, \Omega) \hookrightarrow L^{p}\left(\nu_{\Theta}, \Omega\right) .
$$

Applying Proposition 4.1 in LIONS [16], we obtain that for any $\varepsilon>0$ there exists $c(\varepsilon)>0$ such that for every $u \in V$

$$
\|u\|_{m-1, p, \nu} \leq \varepsilon\|u\|_{m, p, \nu}+c(\varepsilon)\|u\|_{p, \nu \Theta},
$$

i.e.

$$
\begin{aligned}
& \sum_{|\alpha| \leq m-1} \int_{\Omega}\left|D^{\alpha} u(x)\right|^{p} \nu_{\alpha}(x) d x \leq \\
& \leq 2^{p-1} \varepsilon\left[\sum_{|\alpha|=m} \int_{\Omega}\left|D^{\alpha} u(x)\right|^{p} \nu_{\alpha}(x) d x+\sum_{|\alpha| \leq m_{b}-1} \int_{\Omega}\left|D^{\alpha} u(x)\right|^{p} \nu_{\alpha}(x) d x\right]+ \\
& +2^{p-1} c(\varepsilon) \int_{\Omega}|u(x)|^{p} \nu_{\Theta}(x) d x .
\end{aligned}
$$

Consequently, we obtain that for $\varepsilon>0$ small $\left(0<\varepsilon<2^{1-p}\right)$ there is a constant $c_{4}>0$ such that

and hence

$$
\begin{aligned}
\sum_{|\alpha| \leq m-1} \int_{\Omega}\left|D^{\beta} u(x)\right|^{p} \nu_{\alpha}(x) d x & \leq c_{4}\left(\sum_{|\alpha|=m} \int_{\Omega}|D u(x)|^{p} \nu_{\alpha}(x) d x+\right. \\
& \left.+\int_{\Omega}|u(x)|^{p} \nu_{\Theta}(x) d x\right)
\end{aligned}
$$

$$
\begin{aligned}
& \|u\|_{m, p, \nu}^{p}=\sum_{|\alpha| \leq m}\left\|D^{\alpha} u\right\|_{p, \nu_{\alpha}}^{p} \leq c_{5}\left(\sum_{|\alpha|=m}\left\|D^{\alpha} u\right\|_{p, \nu_{\alpha}}^{p}+\right. \\
& \left.+\|u\|_{p, \nu_{\Theta}}^{p}\right)
\end{aligned}
$$

with $c_{5}>0$ independent of $u \in V$. This inequality together with (6.4) yield

$$
\begin{aligned}
& \langle T u, u\rangle=\sum_{|\alpha| \leq m} \int_{\Omega} A_{\alpha}\left(x, u(x), \ldots, D^{m} u(x)\right) \cdot D^{\alpha} u(x) d x \geq \\
& \geq c_{1} \sum_{|\alpha|=m} \int_{\Omega} \nu_{\alpha}(x)\left|D^{\alpha} u(x)\right|^{p} d x+c_{2} \int_{\Omega} \nu_{\Theta}(x)|u(x)|^{p} d x-c_{3} \geq \\
& \geq \min \left(c_{1}, c_{2}\right)\left(\sum_{|\alpha|=m}\left\|D^{\alpha} u\right\|_{p, \nu_{\alpha}}^{p}+\|u\|_{p, \nu_{\Theta}}^{p}\right)-c_{3} \geq \\
& \geq \frac{\min \left(c_{1}, c_{2}\right)}{c_{5}}\|u\|_{m, p, \nu_{\alpha}}^{p}-c_{3}
\end{aligned}
$$

and since $p>1$, we have immediately (6.3) 
Remark 6.2 Instead of (6.4) wr call also use the modificd rocrivity condition

$$
\begin{aligned}
\sum_{|\alpha| \leq m} A_{\alpha}(x, \xi) \xi_{\alpha} & \geq c_{1} \sum_{|\alpha|=m} \nu_{\alpha}(x)\left|\xi_{\alpha}\right|^{p}+ \\
& +c_{2} \omega(x)\left|\xi_{\Theta}\right|^{q}-r_{3}
\end{aligned}
$$

with $q>1$ and a suitable weight function $\omega=\omega(x)$ provided the imbeddings

$$
V \hookrightarrow \hookrightarrow W^{m-1, p}(\nu, \Omega) \hookrightarrow L^{q}(\omega, \Omega)
$$

take place. Indeed, similarly as in Lemma 6.1 we obtain that

$$
\begin{aligned}
\sum_{|\alpha| \leq m-1} \int_{\Omega}\left|D^{\alpha} u(x)\right|^{p} \nu_{\alpha}(x) d x & \leq c_{4}\left[\sum_{|\alpha|=m} \int_{\Omega}\left|D^{\alpha} u(x)\right|^{p} \nu_{\alpha}(x) d x+\right. \\
& \left.+\left(\int_{\Omega}|u(x)|^{q} \omega(x) d x\right)^{\frac{p}{q}}\right]
\end{aligned}
$$

and consequently

$$
\|u\|_{m, p, \nu}^{p} \leq c_{5}\left(\sum_{|\alpha|=m}\left\|D^{\alpha} u\right\|_{p, \nu_{\alpha}}^{p}+\|u\|_{q, \omega}^{p}\right) .
$$

Due to the second imbedding in (6.6) we have

$$
\|u\|_{q, \omega} \leq c_{6}\|u\|_{m-1, p, \nu}=c_{6}\left(\sum_{|\alpha| \leq m-1}\left\|D^{\alpha} u\right\|_{p, \nu_{\alpha}}^{p}\right)^{\frac{1}{p}}
$$

which together with (6.7) imply that the expression

$$
\sum_{|\alpha|=m}\left\|D^{\alpha} u\right\|_{p, \nu_{\alpha}}^{p}+\|u\|_{q, \omega}^{p}=\|\| u\|\|^{p}
$$

defines a norm in $V$ equivalent to $\|\mid u\|_{m, p, \nu}$.

It is

$$
\begin{aligned}
\langle T u, u\rangle & \geq c_{1} \sum_{|\alpha|=m} \int_{\Omega} \nu_{\alpha}(x)\left|D^{\alpha} u(x)\right|^{p} d x+ \\
& +c_{2} \int_{\Omega}|u(x)|^{q} \omega(x) d x-c_{3} \geq \\
& \geq \min \left(c_{1}, c_{2}\right)\left(\sum_{|\alpha|=m}\left\|D^{\alpha} u\right\|_{p, \nu_{\alpha}}^{p}+\|u\|_{q, \omega}^{q}\right)-c_{3} .
\end{aligned}
$$

For $p<q$ it follows from Young's inequality (in the form $a b \leq \frac{a^{r}}{r}+\frac{b^{r^{\prime}}}{r^{\prime}}$ with $r=\frac{q}{p}, a=\left(\int_{\Omega}|u(x)|^{q} \omega(x) d x\right)^{\frac{p}{q}}=$ $\|u\|_{q, w}^{p}, b=1$ ) that

$$
\|u\|_{q, \omega}^{p} \leq \frac{p}{q}\|u\|_{q, \omega}^{q}+c_{7}
$$

Thus we obtain from (6.8) and (6.9) that

$$
\begin{aligned}
\langle T u, u\rangle & \geq \min \left(c_{1}, c_{2}\right)\left(\sum_{|\alpha|=m}\left\|D^{\alpha} u\right\|_{p, \nu}^{p}+\right. \\
& \left.+\frac{q}{p}\|u\|_{q, \omega}^{p}-c_{8}\right) \geq \\
& \geq c_{9}\|\| u \|\left.\right|^{p}-c_{10}
\end{aligned}
$$

which again implies (6.3).

So we have the following result. 
Theorem 6.2 The boundary valuc problem for the differential equation (4.1) in $V$ has for cvery $f \in V^{*}$ at li ast onc weak solution $u \in V$ if ure replace in Theorem 6.1 the condition $(6.1)$ by the rocreivity condition (6.1) or - provided the imbrddings (6.6) hold with $q>p-b y$ the modified cocrcivity condition (6.5)).

Proof Since (6.4) or (6.5) imply (6.3), we have that

$$
\begin{aligned}
& \frac{\left\langle T_{f} u, u\right\rangle}{\|u\|_{m, p, \nu}}=\frac{\langle T u-f, u\rangle}{\|u\|_{m, p, \nu}} \geq \\
& \geq \frac{\langle T u, u\rangle}{\|u\|_{m, p, \nu}}-\frac{\|f\|_{V \cdot\|u\|_{m, p, \nu}}}{\|u\|_{m, p, \nu}}= \\
& =\frac{\langle T u, u\rangle}{\|u\|_{m, p, \nu}}-\|f\|_{V \cdot} \rightarrow \infty \text { if }\|u\|_{m, p, \nu} \rightarrow \infty
\end{aligned}
$$

( $\int \in V^{*}$ is fixed). (onsequently, the condition (6.1) is satisfied for $R>0$ large enough and the assertion follows from Theorem 6.1 .

\section{Remarks and examples}

Remark 7.1 A comparison of the growth conditions mentioned in Section 3 with the growth conditions in [:], Subsection 3.2, gives a good illustration of the broader possibilities offered by the method described here. While the use of spaces $W^{m, p}(\nu, \Omega)$ in [3] with no weights in lower order terms (more precisely, with ' weights $\left.\nu_{\alpha}(x) \equiv 1,|\alpha| \leq m-1\right)$ allowed only changes in the order of the growth (like $\sum_{\kappa_{1} \leq|\beta|<m}\left|\xi_{\beta}\right|^{r_{\beta}}$ with $\left.r_{\beta}>p\right)$, here we can both change the order and introduce weights different from $\nu_{\alpha} \equiv 1$, i.e. introduce degeneration or singularity (see terms like $\sum_{\kappa_{1} \leq|\beta|<m} \omega_{\beta}(x)\left|\xi_{\beta}\right|^{q(\beta)}$ in (3.12)).

Let us consider e.g. the operator $A$ from Example 5.7 in [3] and the Dirichlet problem:

$$
(A u)(x)=(-1)^{m} \sum_{|\alpha|=m} D^{\alpha}\left(\nu(x)\left|D^{\alpha} u(x)\right|^{p-2} D^{\alpha} u(x)\right),
$$

with the weight function $\nu=\nu(x)$ from (1.8). Then we can add, according to [3], lower order terms (say of order $2 m-2$ ) of the form

$$
(-1)^{m-1} \sum_{|\beta|=m-1} D^{\beta}\left(\left|D^{\beta} u(x)\right|^{q-2} D^{\beta} u(x)\right)
$$

with $1<q<2 p-1$ for $1<p<2$. However, the approach described in this paper allows to add e.g. a lower order term of the form

$$
(-1)^{m-1} \sum_{|\beta|=m-1} D^{\beta}\left(\omega(x)\left|D^{\beta} u(x)\right|^{q-2} D^{\beta} u(x)\right),
$$

where the weight function $\omega$ has a similar structure as $\nu$, i.e.

$$
\omega(x)= \begin{cases}1 & \text { for } x_{1} \leq 0 \\ x_{2}^{\lambda_{0}}\left(1-x_{1}\right)^{\gamma_{0}} & \text { for } x_{1}>0, x_{2}>0, \\ \left|x_{2}\right|^{\mu_{0}}\left(1-x_{1}\right)^{\gamma_{0}} & \text { for } x_{1}>0, x_{2}<0\end{cases}
$$

with values $\lambda_{0}, \gamma_{0}, \mu_{0}$ connected with the values $p, q, \lambda, \gamma, \mu$. Roughly speaking it must be

$$
\lambda_{0}>\lambda \frac{q}{p}+\frac{q}{p}-q-1
$$

(with $\lambda$ from (1.8)) and similarly also for $\mu_{0}$ a $\gamma_{0}$.

Remark 7.2 It should be emphasized that the decision about the weight functions $\nu_{\alpha}(x)$ which appear in the definition of the space $W^{m, p}(\nu, \Omega)$ in terms of lower order $(|\alpha| \leq m-1)$ depends on the weight functions $\nu_{\alpha}(x)$ for $|\alpha|=m$ (i. e. appearing at the derivatives of order $m$ ). An important role is played here by Hardy-type inequalities, i.e. inequalities of the form

$$
\left(\int_{\Omega}|v(x)|^{q} \omega(x) d x\right)^{\frac{1}{q}} \leq c\left(\int_{\Omega} \sum_{i=1}^{N}\left|\frac{\partial v}{\partial x_{i}}(x)\right|^{p} \nu_{\imath}(x) d x\right)^{\frac{1}{p}} .
$$


We proceed in the following way: We use inequality (7.4) (say, for $v \in C_{0}^{\prime \infty}(\Omega)$, if we consider the Dirichlet problem) so that we have the imbedding

$$
W_{0}^{1, p}(\nu, \Omega) \hookrightarrow L^{q}(\omega, \Omega) .
$$

Morcover, we assume that the imbedding (7.5) is compact.

Now, we take for $v$ a derivative $D^{\beta} u, \beta$ fixed, $|\beta|=m-1$, and choose for the weight functions $\nu_{\imath}(x)$ on the right--hand side of (7.4) the weight functions $\nu_{\alpha}(x),|\alpha|=m$, appearing in the definition of the space $W^{m, p}(\nu, \Omega)(\operatorname{see}(5.3))$. More precisely, if we denote, for $\beta=\left(\beta_{1}, \beta_{2}, \ldots, \beta_{n}\right),|\beta|=m-1$, by $\beta(\imath)$ the multiindex $\alpha=\left(\alpha_{1}, \alpha_{2}, \ldots \alpha_{N}\right)$ with $\alpha_{3}=\beta_{3}$ for $i \neq j$ and $\alpha_{2}=\beta_{i}+1$, then $|\alpha|=m$ and we choose $\nu_{\imath}(x)=\nu_{\beta(z)}(x)$. Then we can determine the weight function $\omega(x)$ on the left-hand side of $(7.4)$ as $\nu_{\beta}(x)$ if $q=p$ (with $\nu_{\beta}(x)$ appearing in the definition of the space $W^{m, p}(\nu, \Omega)$ ) and as $\omega_{\beta}(x)$ if $q=q(\beta) \neq p$ (with $\omega_{\beta}(x)$ appearing in the definition of the space $H^{m-1, q}(\omega, \Omega)$, see (5.4)).

By this way we determine the weight functions $\nu_{\beta}(x)$ and $\omega_{\beta}(x)$ for $|\beta|=m-1$, and analogously we determine the weight functions appearing at the derivatives of order $m-2$ etc.

Example 7.1 Consider again an operator $A$ whose principal part is of the form (7.1), i.e.

$$
A_{\alpha}(x, \xi)=\nu_{\alpha}(x)\left|\xi_{\alpha}\right|^{p-2} \xi_{\alpha},|\alpha|=m
$$

with $\nu_{\alpha}(x)=\nu(x)$. Let $\Omega$ be a plane domain, $\Omega=\left\{\left(x_{1}, x_{2}\right) ; 0<x_{1}<1,0<\right.$ $\left.<x_{2}<b\right\}$ with $0<b<\infty$, and choose the weight functions $\nu_{\alpha}(x)$ in the form

$$
\nu_{\alpha}(x)=\nu_{\alpha}\left(x_{1}, x_{2}\right)=x_{2}^{\lambda}, \lambda \text { a real number }
$$

In this case the conditions

$$
\nu_{\alpha}^{-\frac{1}{p-1}} \in L_{\text {loc }}^{1}(\Omega), \nu_{\alpha} \in L_{\text {loc }}^{1}(\Omega),|\alpha| \leq m
$$

are obviously fulfilled. Let us consider, for simplicity, the Dirichlet problem. Then the basic space $V$ is just $W_{0}^{m, p}(\nu, \Omega)$ and we can make all considerations for $v$ from the dense set $C_{0}^{\infty}(\Omega)$.

The Hardy inequality

$$
\left(\int_{0}^{b}\left|v\left(x_{1}, x_{2}\right)\right|^{q} x_{2}^{\mu} d x_{2}\right)^{\frac{1}{q}} \leq c\left(\int_{0}^{b}\left|\frac{\partial v}{\partial x_{2}}\left(x_{1}, x_{2}\right)\right|^{p} x_{2}^{\lambda} d x_{2}\right)^{\frac{1}{p}}
$$

holds for all $v \in C_{0}^{\infty}(\Omega)$ with a constatnt $c>0$ independent of $v$ and independent of $x_{1}$, provided

$$
\lambda \neq p-1 \text { and } \mu>\lambda \frac{q}{p}+\frac{q}{p}-q-1
$$

(see Example 6.8 in [18]).

(i) Let us determine the weight functions $\nu_{\beta}(x),|\beta|=m-1$. Take $q=p$ in (7.6), i.e.

$$
\lambda \neq p-1, \mu>\lambda-p
$$

it follows from (7.6) that

$$
\begin{aligned}
& \int_{\Omega}|v(x)|^{p} \omega(x) d x=\int_{0}^{1}\left(\int_{0}^{b}\left|v\left(x_{1}, x_{2}\right)\right|^{p} x_{2}^{\mu} d x_{2}\right) d x_{1} \leq \\
& \leq c^{p}\left(\int_{0}^{1}\left(\int_{0}^{b}\left|\frac{\partial v}{\partial x_{2}}\left(x_{1}, x_{2}\right)\right|^{p} x_{2}^{\lambda} d x_{2}\right) d x_{1}\right) \leq \\
& \leq c^{p} \int_{\Omega}\left[\left|\frac{\partial v}{\partial x_{1}}(x)\right|^{p} \nu(x)+\left|\frac{\partial v}{\partial x_{2}}(x)\right|^{p} \nu(x)\right] d x .
\end{aligned}
$$

This is inequality (7.4) with $\omega(x)=x_{2}^{\mu}, q=p$, which indicates that if we put $v=D^{\beta} u,|\beta|=m-1$, we can choose

Hence, we have

$$
\nu_{\beta}(x)=x_{2}^{\mu} .
$$

$$
\begin{aligned}
& D^{\alpha} u \in L^{p}\left(\nu_{\alpha}, \Omega\right) \text { with } \nu_{\alpha}(x)=x_{2}^{\lambda} \text { for }|\alpha|=m, \\
& D^{\beta} u \in L^{p}\left(\nu_{\beta}, \Omega\right) \text { with } \nu_{\beta}(x)=x_{2}^{\mu} \text { for }|\beta|=m-1,
\end{aligned}
$$

where $\lambda$ and $\mu$ satisfy (7.8). Analogously we can show that 


$$
I^{\gamma} u \in L^{p}\left(\nu_{\gamma}, \Omega\right) \text { with } \nu_{\gamma}(x)=x_{2}^{\delta} \text { for }|\gamma|=m-2
$$

provided

$$
\mu \neq p-1, b>\mu-p,
$$

etc. By this procedure, we construct the space

$$
W^{m, p}(\nu, \Omega)
$$

or more precisely, the space $W_{0}^{m, p}(\nu, \Omega)$. Let us emphasize that the imbedding corresponding to the inequality (7.6) is compact (see Example 7.10 (ii) in [18], where the case $\lambda<p-1$ is discussed; the case $\lambda>p-1$ follows similarly by using Example 6.8 (ii) in [18]). Hence, we have simultaneously the compact imbedding

$$
V=W_{0}^{m, p}(\nu, \Omega) \hookrightarrow \hookrightarrow W_{0}^{m-1, p}(\nu, \Omega) .
$$

(ii) If we would like to determine the space $H^{m-1, p}(\omega, \Omega)$ (satisfying Hypothesis 5.2), i.e. the weight functions $\omega_{\beta}$ for $q \neq p$, we have to differ two cases.

(a) $1<q<p<\infty$. In this case we obtain again a Hardy-type inequality similar to (7.9). Namely, we get

$$
\left(\int_{\Omega}|v(x)|^{q} \omega(x) d x\right)^{\frac{1}{q}} \leq c\left(\int_{\Omega}\left[\left|\frac{\partial v}{\partial x_{1}}(x)\right|^{p}+\left|\frac{\partial v}{\partial x_{2}}(x)\right|^{p}\right] \nu(x) d x\right)^{\frac{1}{p}}
$$

with $\omega(x)=x_{2}^{\mu}$. But now $\mu$ is determined by (7.7), i.e.

$$
\begin{aligned}
& D^{\beta} u \in L^{q(\beta)}\left(\omega_{\beta}, \Omega\right) \text { for }|\beta|=m-1 \\
& \text { with } \omega_{\beta}(x)=x_{2}^{\mu}, \mu>\lambda \frac{q}{p}+\frac{q}{p}-q-1
\end{aligned}
$$

Indeed, using (7.6) and the Hölder inequality with the exponent $r=\frac{p}{q}>1$, we immediately obtain formula (7.10).

Similarly we can determine $\omega_{\beta}(x)$ for $|\beta|=m-2, m-3$ etc.

(b) $1<p<q<\infty$. In this case we cannot use the Hölder inequality. We have to use more complicated procedure described in [18], Subsection 12.13. We will not go into details here. Let us only mention that we again obtain an inequality of the form (7.10) with $\omega(x)=x_{2}^{\mu}$ but now under the additional condition

$$
\frac{1}{p}-\frac{1}{q}>\frac{1}{2}
$$

and with

$$
\mu>\lambda \frac{p}{q} \text {. }
$$

(iii) Due to these investigations, we can consider lower order coefficients of the form

$$
A_{\beta}(x, \xi)=x_{2}^{\mu}\left|\xi_{\beta}\right|^{q-2} \xi_{\beta} \text { for }|\beta|=m-1
$$

and similarly for $|\beta|<m-1$, with $\mu$ depending on $q$ and $p$.

Remark 7.3 In Example 7.1, we investigated the Dirichlet problem on the plane domain $\Omega=] 0,1[\times] 0, b[$ and considered the Hardy inequality (7.6) for functions $v \in C_{0}^{\infty}(\Omega)$. Let us point out that due to the fact that in (7.6) only the integral ] $0, b$ [ for $x_{2}$ is of importance, the same results can be derived if we consider Dirichlet boundary conditions only on the top and the bottom of our rectangle $\Omega$, i.e., for $\left(x_{1}, x_{2}\right) \in \partial \Omega$ with $x_{2}=0$ and $x_{2}=b$.

Moreover, we can consider Dirichlet boundary conditions only on the bottom, $x_{2}=0$ (or only on the top, $x_{2}=b$ ) but then we can consider only values $\lambda$ such that $\lambda<p-1$ (or $\lambda>p-1$ ) instead of $\lambda \neq p-1$.

Let us add one simple example illustrating the modified coercivity condition (6.5).

Example 7.2 Let us consider the Dirichlet problem for the second order equation of the type (4.1), where

$$
(A u)(x)=-\sum_{\imath=1}^{N} \frac{\partial}{\partial x_{\imath}}\left(\nu_{\imath}(x)\left|\frac{\partial u}{\partial x_{\imath}}\right|^{p-2} \frac{\partial u}{\partial x_{\imath}}\right)+\omega(x)|u(x)|^{q-2} u(x)
$$

(here we use "usual" indices instead of multiindices). We have 


$$
\left\{\begin{array}{l}
A_{2}\left(x, \xi_{0}, \xi_{1}, \ldots, \xi_{N}\right)=\nu_{\imath}(x)\left|\xi_{2}\right|^{p-2} \xi_{2} \\
A_{0}\left(x, \xi_{0}, \xi_{1}, \ldots, \xi_{N}\right)=\omega(x)\left|\xi_{0}\right|^{\prime-2} \xi_{0}
\end{array} \text { for } i=1,2, \ldots, N\right.
$$

with $p, q>1$. We use the space $V=W_{0}^{1, p}(\nu, \Omega)$ with $\nu=\left\{\nu_{0}, \nu_{1}, \ldots, \nu_{N}\right\}$ where $\nu_{0}$ is chosen in such a way that the compact imbedding

$$
W_{0}^{1, p}(\nu, \Omega) \smile \hookrightarrow L^{r}\left(\nu_{0}, \Omega\right)
$$

holds. The coercivity condition (6.4) has the form

$$
\begin{gathered}
\sum_{\imath=0}^{N} A_{\imath}\left(x, \xi_{0}, \xi_{1}, \ldots, \xi_{N}\right) \xi_{\imath} \geq c_{1} \sum_{i=1}^{N} \nu_{\imath}(x)\left|\xi_{\imath}\right|^{p}+ \\
+c_{2} \nu_{0}(x)\left|\xi_{0}\right|^{p}-c_{3},
\end{gathered}
$$

i.e., for our special choice (7.11).

$$
\sum_{i=0}^{N} \nu_{\imath}(x)\left|\xi_{\imath}\right|^{p}+\omega(x)\left|\xi_{0}\right|^{q} \geq c_{1} \sum_{i=1}^{N} \nu_{\imath}(x)\left|\xi_{\imath}\right|^{p}+c_{2} \nu_{0}(x)\left|\xi_{0}\right|^{p}-c_{3} .
$$

Since there are no obvious connections between $\omega(x)$ and $\nu_{0}(x), q$ and $p$, we cannot guarrantee that (7.12) is fulfilled for every $\xi \in \mathbf{R}^{N+1}$.

On the other hand, the modified coercivity condition (6.5) is fulfilled. Indeed, it has the form

$$
\begin{gathered}
\sum_{i=0}^{N} A_{\imath}\left(x, \xi_{0}, \xi_{1}, \ldots, \xi_{N}\right) \xi_{\imath} \geq c_{1} \sum_{i=1}^{N} \nu_{\imath}(x)\left|\xi_{\imath}\right|^{p}+ \\
+c_{2} \omega(x)\left|\xi_{0}\right|^{q}-c_{3}
\end{gathered}
$$

and it is obviously satisfied with

$$
c_{1}=c_{2}=1, c_{3} \geq 0 \text {. }
$$

To assure the existence of a weak solution by means of Theorem 6.2, we have to suppose that the Hardytype inequality (7.4) holds for every $u \in V=W_{0}^{1, p}(\nu, \Omega)$, that the corresponding compact imbedding

$$
W_{0}^{1, p}(\nu, \Omega) \hookrightarrow \hookrightarrow L^{q}(\omega, \Omega)
$$

holds, and further that $q>p$.

If we suppose that the following inequality holds:

$$
\int_{\Omega}|u(x)|^{p} \nu_{0}(x) d x \leq c \sum_{i=1}^{N} \int_{\Omega}\left|\frac{\partial u}{\partial x_{i}}(x)\right|^{p} \nu_{1}(x) d x,
$$

then we need not to assume that $p<q$. Indeed, since the right hand side in (7.4) is an equivalent norm in $V$, we have that

$$
\begin{aligned}
\langle T u, u\rangle & =\sum_{i=1}^{N} \int_{\Omega} A_{\imath}(x, u(x), \nabla u(x)) \frac{\partial u}{\partial x_{i}}(x) d x+ \\
& +\int_{\Omega} A_{0}(x, u(x), \nabla u(x)) u(x) d x= \\
& \sum_{i=1}^{N} \int_{\Omega}\left|\frac{\partial u}{\partial x_{i}}(x)\right|^{p} \nu_{1}(x) d x+\int_{\Omega}|u(x)|^{q} \omega(x) d x \geq \\
& \geq c\|u\|_{V}^{p}+\|u\|_{q, \omega}^{q} \geq c\|u\|_{V}^{p},
\end{aligned}
$$

and hence

$$
\lim _{\|u\|_{v \rightarrow \infty}} \frac{\langle T u, u\rangle}{\|u\|_{V}}=\infty
$$

Note that in (7.13), we considered a special family $\nu$ of weight functions, i.e., $\nu=\left\{\nu_{0}, \nu_{1}, \ldots, \nu_{N}\right\}$ with $\nu_{1}=\nu_{2}=\ldots=\nu_{N}$. There are several results concerning the validity of inequality $(7.13)$, called also the wcighted Friedrichs inequality. See e.g., EDMUNDS and OPIC [6] for $\nu_{0}, \nu_{1}$ having singularities or degeneracies only at the boundary and satisfying certain (rather complicated) additional assumptions. 
Acknowledgement: The first two authors have been partially supported by the (irant $A$ gency of ('zech Republic, (irant No. 201/94/00\%, which is here gratefully acknowledged.

\section{References}

(1) F. E. BROWI)ER, Nonluncar clliptic boundary value problems and the generalized topological degrec, Bull. Amer. Math. Soriety 76 (1970), pp. 999 - 1005.

[2] R. ('. BROWN and B. OPIC', Embeddings of weighted Sobolev spaces into spaces of contenuous functıons. Proc. R. Soc. London A (1992) 439, pp. 279 - 296.

[3] P. DRÁBEK, A. KUFNER and F. NI('OLOSI, On the solvability of degeneratcd quasilinear elliptic' fquations of higher order, J. Differential Equations 109 (2) (1994), 325-347.

[4] P. DRÁBEK and F. NICOLOSI, Solubilité des problémes elliptiques dégénérés d'order supérieur a l'ande du théoreme' de Leray-Lions, (.. R. Acad. Sci. Paris 315, Sér. (1992), pp. 689 - 692.

[5] P. DRÁBEK and F. NICOLOSI, Existence of bounded solutions for some degenerated quasilinear clliptıc equations, Annali Mat. Pura Appl. (IV), CLXV (1993), 217-238.

[6] D. E. EDMUNDS and B. OPIC, Weighted Poincaré and Friedrichs inequalities, J. London Math. Soc. (2) 47 (1993), pp. $79-96$.

[7] F. GUGLIELMINO and F. NICOLOSI, Sulle $W$-soluzioni dei problemi al contorno per operatori ellittici degeneri, Ricerche di Matematica 36 (1987), pp. 59 - 72.

[8] F. GUGLIELMINO and F. NICOLOSI, Teoremi di esistenza per i problemi al contorno relativi alle equazioni ellittiche quasilineari, Ricerche di Matematica 37 (1988), pp. 157 - 176.

[9] E. HEWITT and K. STROMBERG, Real and Abstract Analysis, Graduate Texts in Mathematics 25, Springer Verlag Berlin Heidelberg 1975 (Third printing).

[10] A. KUFNER, Weighted Sobolev Spaces, 2nd edition J. Wiley \& Sons, Chichester-New York-BrisbaneToronto-Singapore 1985.

[11] A. KUFNER and S. LEONARDI, Solvability of degenerate elliptic boundary value problems: Another approach, Math. Bohemica 119 (1994), 255-274.

[12] A. KUFNER and B. OPIC, How to define reasonably weighted Sobolev space, Comment. Math. Univ. Carolinae 25 (1984), pp. 537 - 554.

[13] A KUFNER and B. OPIC, The Dirichlet problem and weighted spaces II, Čas. Pěst. Mat. 111(1986), pp. $242-253$.

[14] A. KUFNER and A.-M. SÄNDIG, Some Applications of Weighted Sobolev Spaces, Teubner Texte zur Math. 100, Teubner - Verlag, Leipzig 1987.

[15] S. LEONARDI, On imbedding theorems and Němytskii operators in weighted Sobolev spaces (to appear).

[16] J.-L. LIONS, Équations differentielles operationelles et problémes aux limites, Springer-Verlag, Berlin-Götingen-Heidelberg 1961.

[17] F. NICOLOSI, Soluzioni deboli dei problemi al contorno per operatori parabolici che possono degenerare, Annali Mat. Pura Appl. (4) 125 (1980), pp. 135 - 155.

[18] B. OPIC and A. KUFNER, Hardy-type Inequalities, Pitman Research Notes in Mathematics Series 219, Longman Scientific \& Technical, Harlow 1990.

[19] I. V. SKRYPNIK, Nonlinear Elliptic Boundary Value Problems, 'Teubner Texte zur Math. 91, Teubner-Verlag, Leipzig 1986. 


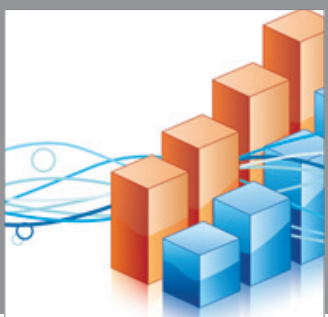

Advances in

Operations Research

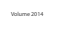

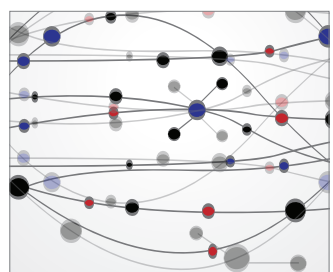

\section{The Scientific} World Journal
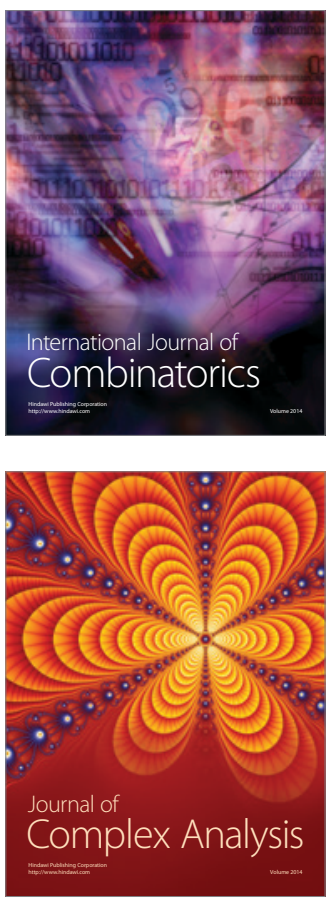

International Journal of

Mathematics and

Mathematical

Sciences
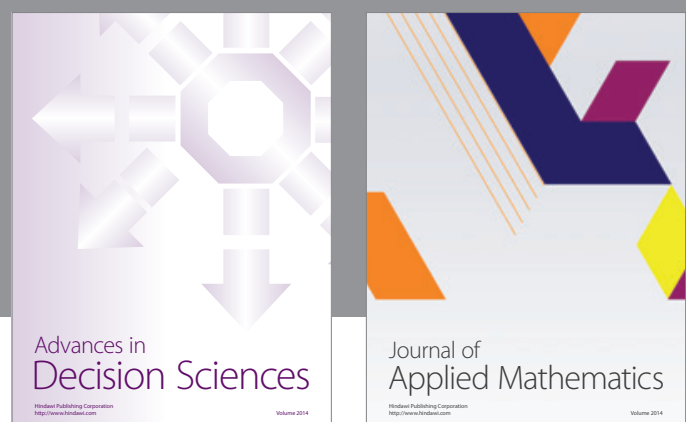

Journal of

Applied Mathematics
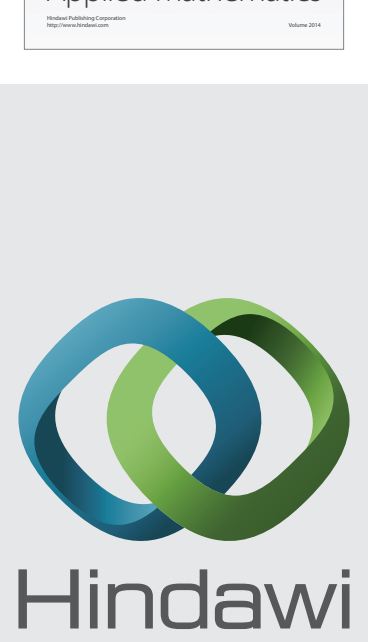

Submit your manuscripts at http://www.hindawi.com
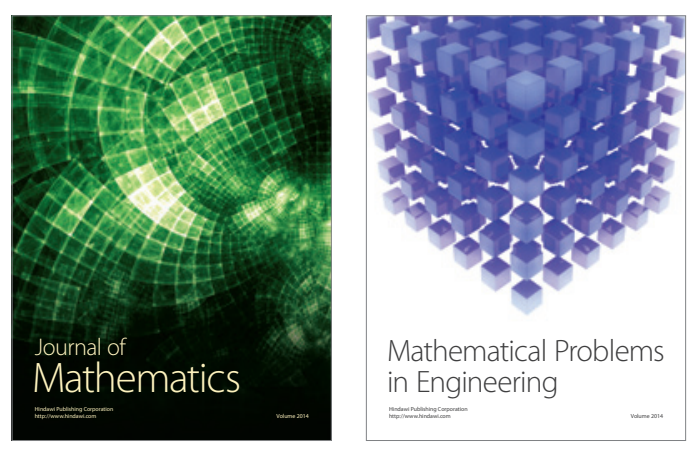

Mathematical Problems in Engineering
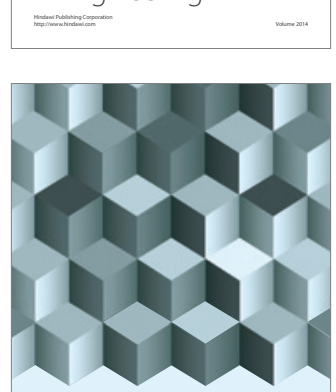

Journal of

Function Spaces
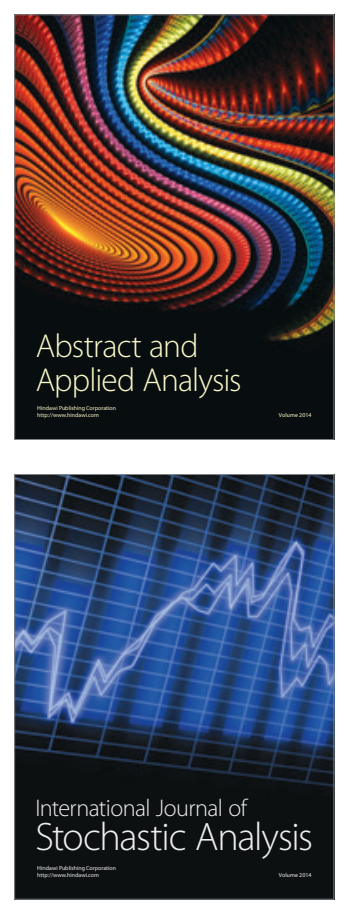

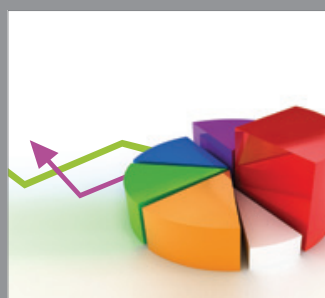

ournal of

Probability and Statistics

Promensencen
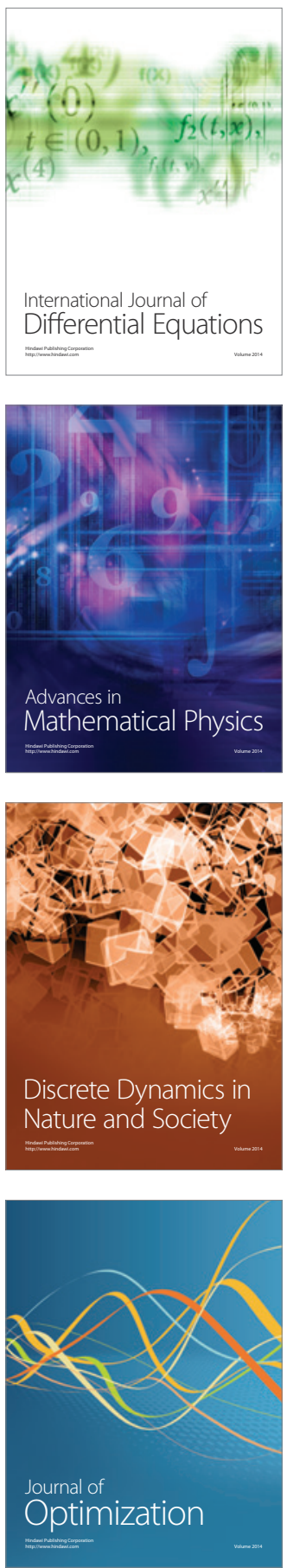